\title{
Pretransplant hepatic malignancy increases risk of de novo malignancy after liver transplantation
}

\author{
Geunhyeok Yang, Shin Hwang, Gil-Chun Park, Chul-Soo Ahn, Ki-Hun Kim, Deok-Bog Moon, Tae-Yong Ha, Gi-Won Song, \\ Dong-Hwan Jung, Sung-Gyu Lee
}

Department of Surgery, Asan Medical Center, University of Ulsan College of Medicine, Seoul, Korea

Background: Hepatocellular carcinoma (HCC) recurrence and development of de novo malignancy (DNM) after liver transplantation (LT) are the major causes of late recipient death.

Methods: We analyzed the incidence of extrahepatic DNM following living donor LT according to the status of pretransplant hepatic malignancy. We selected 2,076 adult patients who underwent primary LDLT during 7 years from January 2010 to December 2016.

Results: The pretransplant hepatic malignancy group $(n=1,012)$ showed 45 cases $(4.4 \%)$ of the following extrahepatic DNMs: posttransplant lymphoproliferative disease (PTLD) in 10; lung cancer in 10; stomach cancer in six; colorectal cancer in five; urinary bladder cancer in three; and other cancers in 11. The pretransplant no hepatic malignancy group $(n=1064)$ showed 25 cases (2.3\%) of the following extrahepatic DNMs: colorectal cancer in three; stomach cancer in three; leukemia in three; lung cancer in three; PTLD in two; prostate cancer in two; and other cancers in nine. Incidences of extrahepatic DNM in the pretransplant hepatic malignancy and no hepatic malignancy groups were as follows: $1.1 \%$ and $0.5 \%$ at 1 year, $3.2 \%$ and $2.0 \%$ at 3 years, $4.6 \%$ and $2.5 \%$ at 5 years, and $5.4 \%$ and $2.8 \%$ at 8 years, respectively $(P=0.006)$. Their overall patient survival rates were as follows: $97.3 \%$ and $97.2 \%$ at 1 year, $91.6 \%$ and $95.9 \%$ at 3 years, $89.8 \%$ and $95.4 \%$ at 5 years, and $89.2 \%$ and $95.4 \%$ at 8 years, respectively $(P<0.001)$. Pretransplant hepatic malignancy was the only significant risk factor for posttransplant extrahepatic DNM.

Conclusions: Our results suggest that patients who had pretransplant hepatic malignancy be followed up more strictly because they have a potential risk of primary hepatic malignancy recurrence as well as a higher risk of extrahepatic DNM than patients without pretransplant hepatic malignancy.

Corresponding author: Shin Hwang

E-mail: shwang@amc.seoul.kr

\footnotetext{
(C) The Korean Society for Transplantation

This is an Open Access article distributed under the terms of the Creative Commons Attribution Non-Commercial License (http://creativecommons.org/licenses/by-nc/4.0/) which permits unrestricted non-commercial use, distribution, and reproduction in any medium, provided the original work is properly cited.
} 